\title{
Monte-Carlo Simulations of the Suzaku-XRS Residual Background Spectrum
}

\author{
E. Perinati • C.A. Kilbourne $\cdot$ L. Colasanti • \\ S. Lotti • C. Macculi • L. Piro • T. Mineo • \\ K. Mitsuda • A. Bonardi • A. Santangelo
}

Received: 29 July 2011 / Accepted: 22 February 2012 / Published online: 8 March 2012

(C) Springer Science+Business Media, LLC 2012

\begin{abstract}
Cryogenic micro-calorimeters are suitable to detect small amounts of energy deposited by electromagnetic and nuclear interactions, which makes them attractive in a variety of applications on ground and in space. The only X-ray microcalorimeter that operated in orbit to date is the X-Ray Spectrometer on-board of the Japanese Suzaku satellite. We discuss the analysis of the components of its residual background spectrum with the support of Monte-Carlo simulations.
\end{abstract}

Keywords X-ray spectroscopy $\cdot$ Background $\cdot$ Monte-Carlo simulations

\section{Introduction}

The X-Ray Spectrometer (XRS) [1] on-board of the Japan/US Suzaku space mission was the first (and unique, to date) X-ray micro-calorimeter detector placed in orbit.

E. Perinati $(\bowtie) \cdot$ A. Bonardi · A. Santangelo

IAAT-Institut für Astronomie und Astrophysik, Universität Tübingen, 72076 Tübingen, Germany

e-mail: emanuele.perinati@uni-tuebingen.de

E. Perinati $\cdot$ T. Mineo

INAF-Istituto di Astrofisica Spaziale e Fisica Cosmica di Palermo, 90146 Palermo, Italy

C.A. Kilbourne

NASA —Goddard Space Flight Center, Greenbelt, MD 20771, USA

L. Colasanti $\cdot$ S. Lotti $\cdot$ C. Macculi $\cdot$ L. Piro

INAF_-Istituto di Astrofisica Spaziale e Fisica Cosmica di Roma, 00133 Roma, Italy

L. Piro

Astronomy Department, Faculty of Science, King Abdulaziz University, P.O. Box 80203,

Jeddah 21589, Saudi Arabia

K. Mitsuda

JAXA-Institute of Space and Astronautical Science, 229-8150 Sagamihara, Japan 
Unfortunately, due to a premature loss of the stored liquid helium XRS worked only for three weeks, actually without seeing the sky (gate valve closed). However, the data collected in that temporal window provide information about the instrumental non $X$ ray background $(N X B)$ induced by the space radiation environment. Therefore, in the perspectives of future missions equipped with micro-calorimeters, it is interesting to achieve a full understanding of the nature of the XRS residual background spectrum. Suzaku was launched in 2005 into a Low Earth Orbit (LEO) at $~ 600 \mathrm{Km}$ altitude with $\sim 30$ deg inclination. The XRS instrument developed at the NASA Goddard Space Flight Center is an array of $6 \times 6$ pixels. Each pixel in the array is made of a HgTe absorber $624 \times 624 \times 8.8 \mu \mathrm{m}$ kept in thermal contact with a some smaller ionimplanted Si thermometer. In order to reduce as much as possible the number of Xray-like events produced by the interaction of particles from the space environment, XRS was provided with a $10 \times 10 \times 0.5 \mathrm{~mm}$ Si-based ionization anti-coincidence detector, placed $630 \mu \mathrm{m}$ behind the array. However, the spectrum of events in the anti-coincidence was not recorded, a flag was assigned to micro-calorimeter pulses occurring in coincidence with an anti-co pulse. A threshold was set on the anti-co pulses, but there was no evaluation of their pulse heights.

\section{XRS Residual Background Spectrum}

Figure 1 shows the XRS residual background spectrum $50 \mathrm{eV}$-binned. Through the anti-coincidence $99.9 \%$ of the X-ray-like events generated directly by primary particles are rejected [1]. Therefore, there are only 207 counts left between $0.1-12 \mathrm{keV}$, collected by 30 pixels in $37100 \mathrm{sec}$ between South Atlantic Anomaly (SAA) crossings. Normalization to the geometrical area gives a rate of $\sim 0.05 \mathrm{cts} \mathrm{cm}^{-2} \mathrm{sec}^{-1}$. Since we expect, on average, $\sim 4000$ primary interactions over the array area in $37100 \mathrm{sec}$, the left events should to be caused mostly by secondary emission from the structures surrounding XRS. Even though the statistics of the collected data is poor, we can make some considerations on this spectrum: below $1 \mathrm{keV}$ counts are concentrated in few features, the explanation of their origin is not obvious. At $\sim 1.5 \mathrm{keV}$ a feature is visible which could be likely attributed to the emission of $\mathrm{K}$-fluorescence and Auger electrons from the $\mathrm{Al}_{2} \mathrm{O}_{3}$ board on which the microcalorimeter is mounted. Also it seems reasonable to assume that the feature at

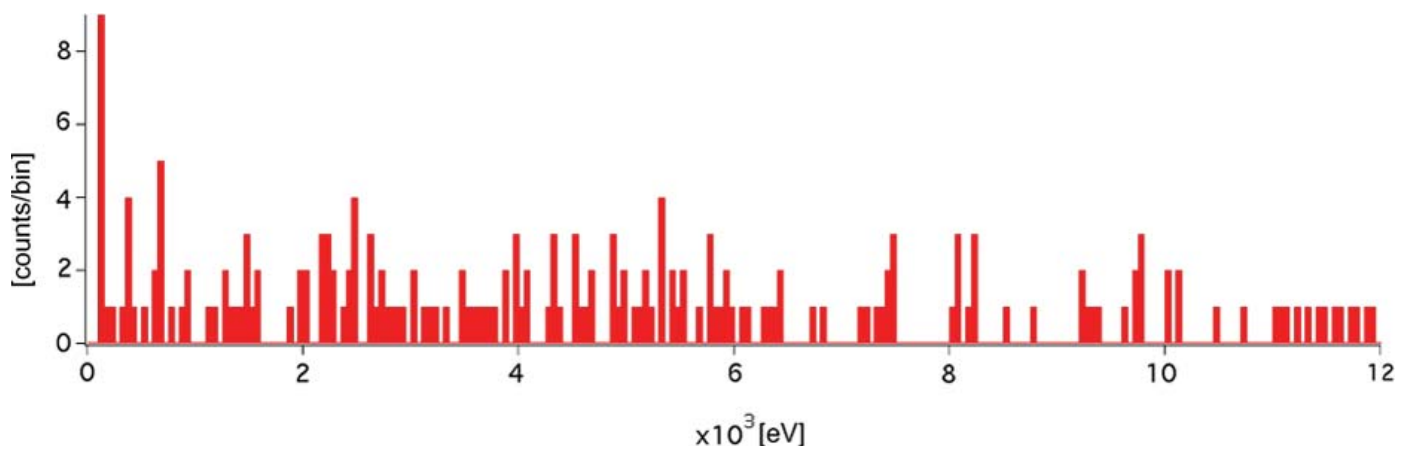

Fig. 1 (Color online) The residual NXB spectrum observed by XRS 
9.7 $\mathrm{keV}$ is originated from the L-fluorescence of the Au coating of the detector box. Other pseudo-features in the spectrum could be related to K-fluorescence of $\mathrm{Cr}(\sim 5.4 \mathrm{keV})$ and $\mathrm{Ni}(\sim 7.5 \mathrm{keV})$, respectively. In principle, such elements are not known to be present in the XRS detector box, however we remark that they are components of stainless steel, and that $\mathrm{Cr}$ and $\mathrm{Ni}$ fluorescence lines are visible also in the NXB spectrum of XMM-EPIC CCDs. In order to explore with greater detail the XRS residual spectrum, we implemented a model of XRS and performed MonteCarlo simulations.

\section{XRS Model}

We used GEANT4 9.4 to simulate XRS in LEO. We adopted a simplified description of the distribution of masses surrounding XRS assuming a multi-shell geometry with spherical symmetry. The outermost shell (Al, thickness $=7 \mathrm{~mm}$ ) simulates the Suzaku optical bench. Inside this volume we placed a second shell (Al, thickness $=5 \mathrm{~mm})$ simulating the cryostat wall. The innermost shell $(\mathrm{Cu}+\mathrm{Au}$ coating, thickness $=2 \mathrm{~mm}$ ) simulates the XRS detector box. Inside the detector box we placed XRS and the anti-coincidence, modeled as two layers made of $\mathrm{HgTe}$ and $\mathrm{Si}$, respectively. We assigned a $16 \mathrm{keV}$ threshold to the anti-coincidence, as in the real case. Particles are shot isotropically from the boundary of the outermost shell.

The LEO radiation environment presents different components, with cosmic and atmospheric origin. However, the cosmic particles to be considered for possible contribution to the NXB are basically protons and electrons, since most cosmic photons (CXB) have energy below $20 \mathrm{keV}$ and are fully absorbed by the optical bench structure. The atmospheric albedo radiation includes protons, electrons, positrons, neutrons and high energy photons. Assuming the fluxes described in [2-4] we calculated in the range $10 \mathrm{MeV}-100 \mathrm{GeV}$ a flux $\sim 0.3 \mathrm{~cm}^{-2} \mathrm{sec}^{-1}$ for protons, $\sim 0.1 \mathrm{~cm}^{-2} \mathrm{sec}^{-1}$ for electrons, $\sim 0.2 \mathrm{~cm}^{-2} \mathrm{sec}^{-1}$ for positrons; in the range $100 \mathrm{meV}-100 \mathrm{GeV}$ we calculated a flux $\sim 0.7 \mathrm{~cm}^{-2} \mathrm{sec}^{-1}$ for neutrons; in the range $3 \mathrm{keV}-6 \mathrm{MeV}$ we calculated a flux $\sim 9 \mathrm{~cm}^{-2} \mathrm{sec}^{-1}$ for high energy photons. For charged particles these estimates include both the cosmic and albedo components, and are obtained assuming an average cut-off at $8.8 \mathrm{GeV}$ for cosmic protons and electrons due to the geo-magnetic shielding.

\section{Results and Discussion}

We set a diameter $D=22 \mathrm{~mm}$ for the outermost shell and shot particles isotropically from a distance $r=D$. For each type of particle, we derived from the adopted flux the number of shots onto the shell in $37100 \mathrm{sec}$. The residual background spectrum simulated using GEANT4 9.4 (Fig. 2) reproduces quite well that measured by XRS. However, the total number of residual counts between $0.1-12 \mathrm{keV}$ obtained in $37100 \mathrm{sec}$ is 158 , corresponding to $\sim 75 \%$ of the residual counts observed by XRS. As for the spectral shape, it is really hard to try a comparison with real data due to the low statistics, however it seems that the accordance of the simulated spectrum with 
Fig. 2 Simulated XRS NXB spectrum in $37100 \mathrm{sec}$

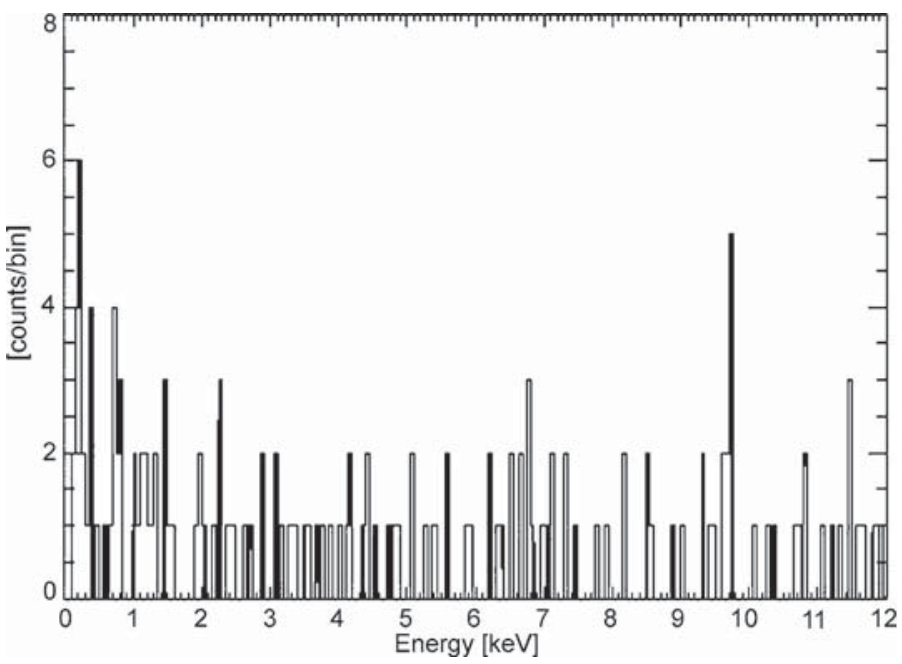

Fig. 3 Simulated XRS NXB spectrum in $10 \times 37100 \mathrm{sec}$

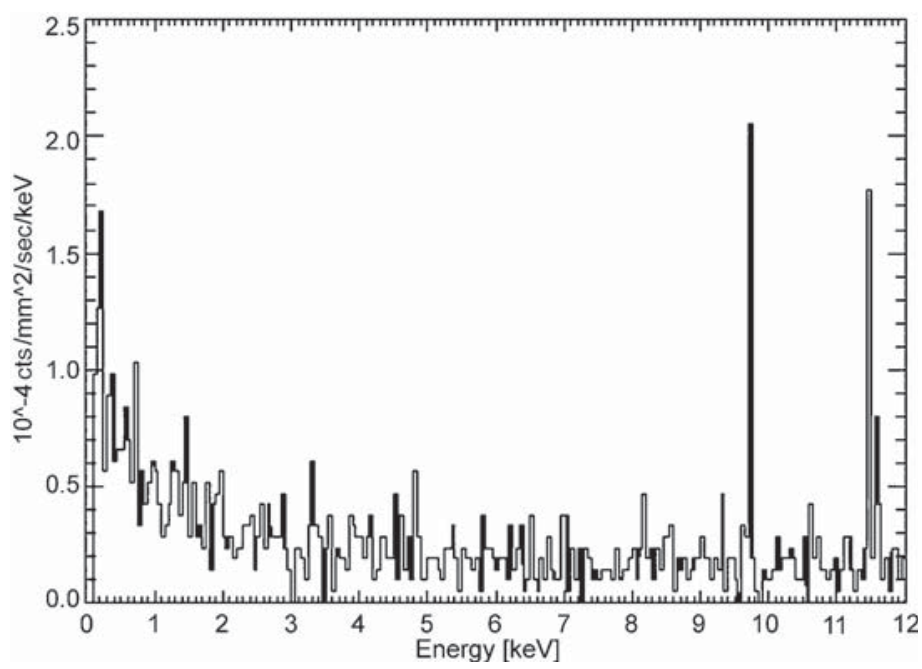

the observed one is good in the lower energy range (below $2 \mathrm{keV}$ ) and higher energy range (above $8 \mathrm{keV}$ ), even though in the simulated spectrum we obtain a number of $\mathrm{Au}$ fluorescences at $\sim 9.7 \mathrm{keV}$ and $\sim 11.4 \mathrm{keV}$ in excess with respect to the counts observed at those energies; differences in the population of the channels are more evident between $2 \mathrm{keV}$ and $8 \mathrm{keV}$, and this could be due to something missing in the model, or simply to the poor statistics.

The analysis of the simulated output indicates that the NXB is induced by the structures closest to XRS: $\sim 80 \%$ of the NXB is produced by knock-on electrons ejected by the Au coating of the detector box and the alumina XRS board, while $\sim 20 \%$ consists of Bremsstrahlung and fluorescence photons emitted from such structures. The $\sim 0.9 \mathrm{cts} \mathrm{cm}^{-2} \mathrm{sec}^{-1}$ count-rate measured by the anti-coincidence is correctly reproduced as well in the simulated data, giving $\sim 0.84 \mathrm{cts} \mathrm{cm}^{-2} \mathrm{sec}^{-1}$. We show also how the simulated spectrum looks at higher statistics on a time-scale 10 times longer (Fig. 3), where the L-fluorescences of Au become stronger and dominate the spectrum. Indeed, simulations indicate that the spectral evolution is towards a continuum made mostly of secondary knock-on electrons and Bremsstrahlung photons plus strong Au fluorescences. 
It is worth to stress that micro-calorimeters measure energy deposited anywhere in the detector, there is no dead layer like there is in a CCD and pulses of the heat sink temperature appear as signals [5, 6]. The XIS instrument on Suzaku has 4 CCDs, one of them is a BI-CCD, thinner that the others and with extremely reduced dead layer: the NXB observed by this BI-CCD is similar to that seen by XRS, while the NXB observed by the three FI-CCDs is $\sim 3$ times lower.

\section{Conclusion}

In conclusion, considering that our simulations are based on a number of approximations in modeling the geometry and the radiation environment and that the statistics of the available observed data is poor, the simulated XRS spectrum seems to reproduce with good accordance the observed one, and therefore we are confident that the interpretation of the recorded data done through GEANT4 is reliable. However, the integral number of counts in the simulations is still $\sim 25 \%$ less than that observed. This could be due to the assumptions and approximations on the input fluxes, to possible activation and/or non-prompt emission induced by SAA passages and to some details not included yet in the geometry, therefore we are still working to improve the model to obtain better accuracy.

Acknowledgements We acknowledge ASI under contract I/035/10/0.

\section{References}

1. R.L. Kelley et al., Publ. Astron. Soc. Jpn. 59, 77 (2007)

2. T. Mizuno et al., Astrophys. J. 614, 1113 (2004)

3. D. Gruber et al., Astrophys. J. 520, 124 (1999)

4. T.W. Armstrong et al., J. Geophys. Res. 78, 2715 (1973)

5. C.K. Stahle et al., Nucl. Instrum. Methods Phys. Res. A 520, 472 (2004)

6. C.A. Kilbourne et al., Nucl. Instrum. Methods Phys. Res. A 559, 620 (2006) 